\title{
Do Our Pharmacy Standards Promote Integrated Care?
}

\author{
Christine M Bond and Yvonne Hopf
}

$\mathrm{H}$

ealth care should be clinically effective, cost-effective and

safe. Worldwide, reforms are in progress to cope with the rising costs of health care resulting from demographic changes and medical advances. Two approaches are commonly adopted to address these challenges: utilizing the skills of the wider health care workforce and transferring the balance of care from secondary to primary care settings. At the same time, it is accepted that patients should be partners in decision-making about their care, which leads to patient empowerment and better health outcomes. These service reforms have been challenged because of concerns over safety, and continuity of care may have suffered. Patients complain of having to repeatedly tell an increasing number of different professionals the same details of their medical (and social) situations.

A relatively recent concept designed to address some of these concerns is integrated care, defined by the World Health Organization as "a concept bringing together inputs, delivery, management and organisations of service related to diagnosis, treatment, care, rehabilitation and health promotion. Integration is a means to improve services in relation to access, quality, user satisfaction and efficiency." Integrated care is not a single entity but a multidimensional philosophy of care, including vertical integration across settings and horizontal integration within teams.

Those with the most complex health care needs are most likely to benefit from integrated care. Data from the United Kingdom show that 4 out of 5 people 75 years of age or older take a prescription medicine, and $36 \%$ are taking 4 or more. ${ }^{2}$ Adverse events associated with medicines are increasingly common because of drug interactions from polypharmacy regimens, unnoticed contraindications to prescribed medicines due to comorbidities, and the physiological changes associated with aging. All health care professionals need to be aware of the changing balance of risks and benefits of medicine use in patients with complex needs, and pharmacists, as experts in medicines, are best placed to advise on safe and effective medicine management.

Pharmacists know how integral their profession is to ensuring safe, clinically effective, and cost-effective medicine use.
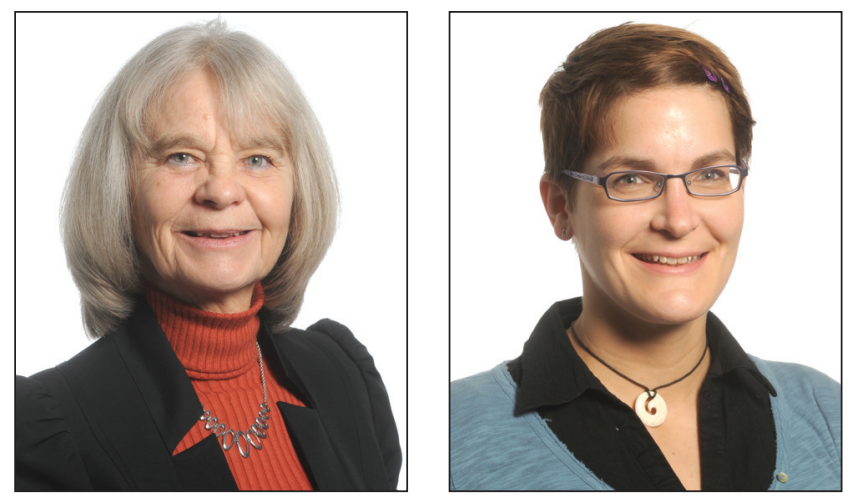

However, despite pharmacy's inalienable right to be represented in the core health care team, delivering integrated care, most of the initiatives related to integrated care centre on physicians' roles and practices; involvement of members of the wider health care team is commonly limited to nursing.

To make the case to our colleagues who are not pharmacists, consider the following 3 examples. A recent large study conducted in Scotland showed that ward pharmacists identified and resolved prescribing errors in the drug charts of $36 \%$ of patients. $^{3}$ On average, $7.5 \%$ of the prescriptions written by doctors contained an error. ${ }^{3}$ Integrating pharmacists earlier into the prescribing process and enabling them to apply their traditional skills of prescription checking at an earlier stage would pre-empt such errors and avoid the risk of them being undetected because of a lack of pharmacy resources to cover all wards. Yet a common way to reduce health care costs is by freezing vacant positions, and so pharmacy teams are depleted at the very time pharmacists are needed most.

The second example builds on the first. Most errors occur either at admission or upon discharge. Although medicine reconciliation is recognized widely as one way of reducing errors in practice, many challenges remain. Patients, especially those with unscheduled admissions, may not always be able to clearly communicate their current medications. In addition, access to "emergency care records" (a password-protected electronic dataset with core medical information on every patient in Scotland, accessible by authorized doctors and pharmacists 
providing unscheduled care) is not always possible, and pharmacists are not always present at the point of admission.

Pilot studies in Scotland have demonstrated the benefit of electronically sharing, between community and hospital pharmacists, information about a patient's medications at both admission and discharge. Patients have been willing to consent to this use of their data, and pharmacists believe medication safety incidents have been averted. This is an excellent example of integration of care across settings with the patient at its heart.

The third example is relevant to both service redesign and extended roles. Pharmacist prescribing has now been introduced in Canada and the United Kingdom and is especially well established in Scotland. In some hospitals, the majority of pharmacists are prescribing, often in clinical areas where specialist understanding of medications is required (e.g., oncology and cystic fibrosis). Anecdotal and research evidence suggests that prescribing by pharmacists is well regarded and effective. In a study of general practice-based pharmacist prescribing for patients with chronic pain, pain outcomes were significantly better in the group with pharmacist prescribing than in the usual care group. ${ }^{4}$ However, despite these endorsements, financial constraints are limiting wider implementation. Instead, prescribing is delegated to specialist nurses, who have less formal training in therapeutics.

Much of what pharmacists do is governed by professional standards, and to conclude this article, we reflect on how such standards promote integrated care.

The Canadian standards for professional hospital pharmacy practice $^{5}$ contain many relevant statements to support integrated care, including 2.4 ("Responsible for continuous pharmaceutical care and seamless care of patients"), 2.8 ("Collaborates with patients to assess needs, establish mutual goals and develop and implement a care plan"), 2.9 ("Collaborates with other health care providers"), and 4.1 ("Develops and sustains collaborative partnerships with patients, patient groups, other healthcare professionals ...").

The UK standards ${ }^{6}$ reflect similar aspirations. One of their 10 listed dimensions of care is "integrated transfer of care". Given that pharmacists already have an appropriate framework in place, what more should be done to ensure that this framework is translated into a practice model that allows pharmacists to play their part in delivering integrated care? Four approaches are suggested.

First, there is a need to create and disseminate a greater body of research evidence. A single study will not change practice. Studies such as those summarized above should trigger further work extending the evidence to different geographic and clinical contexts. Second, further and meaningful joint undergraduate and postgraduate training is needed, to increase mutual interprofessional understanding and respect. Third, in jurisdictions where pharmacists do not have access to patients' complete medical records, such access should be established, to enable pharmacists to provide the best pharmaceutical care, informed by previous medical history and management. Finally, all pharmacists must lobby policy-makers to ensure that the skills of the profession are fully recognized and integrated into health care planning. Hospital pharmacists should certainly rejoice in the undoubted progress of the past decade, but much remains to be done.

References

1. Gröne O, Garcia-Barbero M. Trends in integrated care-reflections on conceptual issues. Copenhagen (Denmark): World Health Organization; 2002. Publ no. EUR/02/5037864.

2. Quality and outwork framework: NHS employers 2012. In: Scottish Government prescription for excellence: a vision and action plan. Edinburgh (UK): Scottish Government; 2013.

3. Ryan C, Ross S, Davey PG, Duncan EM, Fielding S, Francis J, et al. Prevalence and causes of prescribing errors: the PRescribing Outcomes for Trainee doctors Engaged in Clinical Training (PROTECT) study. PLOS $O N E$. In press.

4. Bruhn H, Bond CM, Elliott AM, Hannaford PC, Lee AJ, McNamee P, et al. Pharmacist-led management of chronic pain in primary care: results from a randomised controlled exploratory trial. BMJ Open. 2013;3(4):e002361.

5. Professional hospital pharmacy practice: standards. Ottawa (ON): Canadian Society of Hospital Pharmacists; 2003.

6. Professional standards for hospital pharmacy services: optimising patient outcomes from medicines. London (UK): Royal Pharmaceutical Society; 2012 Jul [cited 2013 Nov 22]. Available from: www.rpharms.com/support-pdfs/rps--professional-standards-for-hospital-pharmacy.pdf

Christine M Bond, BPharm, PhD, MEd, is with the Pharmacy, Centre of Academic Primary Care, University of Aberdeen, Foresterhill, Aberdeen, Scotland. She is also an Associate Editor with the CJHP.

Yvonne Hopf, MPharmS, MSc, PhD, is with the Centre of Academic Primary Care, University of Aberdeen, Foresterhill, Aberdeen, Scotland. She is also a Clinical Pharmacist with the Royal Aberdeen Infirmary.

Competing interests: Christine Bond has received reimbursement from Wiley for travel expenses to attend professional meetings and conferences in her role as Editor in Chief of the International Journal of Pharmacy Practice, as well as travel reimbursement from the Canadian Institutes of Health Research to contribute to "Best Brains Exchanges 2012". For research activities outside the scope of this article, she has received multiple research grants (paid to her institution) from European, UK, and Scottish research councils and charities, as well as a grant from Novartis. Yvonne Hopf received a National Health Service Applied Programme Grant and a postdoctoral fellowship from the Chief Scientist Office of Scotland for work outside the scope of this article.

\section{Address correspondence to:}

Professor Christine M Bond

Pharmacy

Centre of Academic Primary Care

Room 1.123

Polwarth Building West Block

Foresterhill, Aberdeen AB25 2ZD

Scotland

e-mail: c.m.bond@abdn.ac.uk 